\title{
Traduire
}

Revue française de la traduction

\section{La traduction assermentée en Espagne, en France et en République tchèque}

Jaromír Kadlec

\section{OpenEdition}

Journals

Édition électronique

URL : http://journals.openedition.org/traduire/952

DOI : $10.4000 /$ traduire.952

ISSN : 2272-9992

\section{Éditeur}

Société française des traducteurs

\section{Édition imprimée}

Date de publication : 1 décembre 2017

Pagination : 77-83

ISSN : 0395-773X

\section{Référence électronique}

Jaromír Kadlec, «La traduction assermentée en Espagne, en France et en République tchèque »,

Traduire [En ligne], 237 | 2017, mis en ligne le 01 décembre 2017, consulté le 29 juin 2019. URL : http:// journals.openedition.org/traduire/952 ; DOI : 10.4000/traduire.952 


\title{
La traduction assermentée en Espagne, en France et en République tchèque
}

\author{
Jaromír Kadlec
}

\section{Cadre légal}

En 1977, l'Espagne a adopté le règlement de l'Office d'Interprétation des langues du ministère des Affaires étrangères, approuvé par le décret royal 255/1977 du 27 août. Depuis, le décret royal a subi plusieurs modifications (en 1987, en 1992, en 1996 et en 2009). Outre ce décret, le ministère des Affaires étrangères et de la Coopération a adopté plusieurs ordonnances fixant les conditions de nomination des traducteurs-interprètes jurés. Mentionnons notamment l'ordonnance du ministère des Affaires étrangères et de la Coopération 2125/2014 du 6 novembre. Pour devenir traducteur-interprète juré en Espagne, il faut passer un examen. Entre 1996 et 2009, le décret royal permettait de nommer des diplômés en traduction et interprétation ayant obtenu 24 crédits en traduction juridique et économique ou 16 crédits en interprétation juridique et économique, ce qui a suscité un conflit ouvert entre les universités et les associations professionnelles qui défendaient les intérêts de leurs membres et refusaient d'accepter que les jeunes diplômés sans expérience professionnelle soient automatiquement nommés interprètes jurés. De fait, la dernière modification du décret royal de 2009 a supprimé cette possibilité suite à la disparition de l'ancienne licence espagnole. Dans les nouveaux programmes d'études, le nombre de crédits a diminué de 300 à 240 : il y a moins de matières à option et, par conséquent, moins de cours spécialisés en traduction juridique. Les diplômés de 2015 étaient les derniers à pouvoir demander leur nomination sans avoir à passer d'examens spécifiques. La modification du décret royal, adoptée en 2009, a apporté également un autre changement majeur : depuis 2009, l'interprète juré est en effet nommé traducteur-interprète juré, car il est non seulement chargé des interprétations, mais aussi des traductions. Selon l'Ordonnance du ministère des Affaires étrangères et de la Coopération du 6 novembre, les candidats doivent être majeurs, de nationalité espagnole ou avoir la nationalité d'un autre pays de l'Union européenne ou de l'Espace économique européen et être titulaires d'une Licence ou d'un Master espagnol ou étranger, reconnu par le ministère de l'Éducation, de la Culture et des Sports. Jusqu'en 1988, les candidats n'avaient qu'une seule épreuve à 
effectuer, à savoir la traduction, avec dictionnaire, d'un texte juridique ou économique en espagnol. Entre 1988 et 1996, l'épreuve comprenait deux parties : la traduction d'un texte général en espagnol sans dictionnaire et la traduction d'un texte juridique ou économique en espagnol avec dictionnaire. Aujourd'hui, l'examen est composé de trois épreuves. La première consiste en un test grammatical et terminologique. Seuls les candidats qui le réussissent peuvent passer la suivante, elle-même constituée de trois parties : la traduction d'un texte de caractère général en espagnol sans dictionnaire, la traduction d'un texte de caractère général de l'espagnol en langue étrangère sans dictionnaire et la traduction d'un texte juridique ou économique en espagnol, avec dictionnaire. Enfin, la troisième épreuve, cette fois orale, est une interprétation consécutive qui, si le jury l'estime nécessaire, peut s'accompagner d'un entretien avec l'intéressé en langue étrangère. Pour harmoniser les règles relatives à l'évaluation des traductions écrites, l'Office d'Interprétation des langues a publié en 2011 les critères applicables à la correction des différentes parties de l'examen. Par ailleurs, les honoraires des traducteurs/interprètes sont libres, conformément à l'article 11 du décret royal. La réglementation espagnole relative à la traduction et à l'interprétation jurées porte surtout sur la fixation des normes d'accès au métier. Mais dès que le traducteur-interprète accède au statut de juré, il n'existe pratiquement aucune règle définissant un exercice correct et uniforme de son métier.

La législation et les règles d'accès au métier de traducteur et/ou interprète assermenté en France diffèrent largement du modèle espagnol. L'expert traducteur et/ou interprète agréé fait partie, avec d'autres professionnels, du groupe des experts judiciaires. Les premières réglementations concernant l'expertise datent du xıxe siècle, mais la codification principale a été établie par la loi n 71-498 du 29 juin 1971 définissant le métier d'expert. Cette loi précise également les rôles et fonctions des experts en établissant les premières modalités d'inscription. Elle a été ensuite détaillée et appliquée par le décret $n^{\circ}$ 74-1184 du 31 décembre 1974. II s'agit de la première reconnaissance de la profession d'interprète assermenté. En France, il n'y a aucun examen officiel ni pour les experts traducteurs ni pour les interprètes qui appartiennent à la même catégorie d'experts. II n'y a donc aucune règle qui harmonise le choix des experts traducteurs et/ou interprètes. Ce dernier relève de la Cour compétente chargée d'étudier le dossier soumis par le candidat. Toute personne physique souhaitant devenir traducteur et/ou interprète expert doit remplir les conditions légales générales qui s'appliquent à tous les experts judiciaires. Les conditions d'inscription sur la liste d'experts près d'une Cour d'appel sont fixées dans l'article 2 du décret $n^{\circ}$ 2004-1463 du 23 décembre 2004 relatif aux experts judiciaires modifié par le décret $n^{\circ}$ 2007-1119 du 19 juillet 2007. Les conditions pour les personnes morales sont différentes et sont fixées par l'article 3 du décret $n^{\circ}$ 2004-1463 du 23 décembre 2004 relatif aux experts judiciaires, modifié par le décret n²007-1119 du 19 juillet 2007. Selon l'article 6 du décret $n^{\circ}$ 2004-1463 du 23 décembre 2004, le candidat qui fait sa première demande d'inscription pour une durée initiale de trois ans sur une liste dressée par une Cour d'appel doit envoyer son dossier avant le 1er mars de chaque année à l'attention du procureur de la République près du Tribunal de grande instance du ressort de la Cour d'appel. 
Le candidat peut faire figurer une ou plusieurs rubriques et spécialités, doit indiquer les diplômes obtenus, les travaux scientifiques, techniques ou professionnels réalisés, les différentes fonctions occupées et les moyens et installations dont il dispose. Il doit également justifier son expertise dans la spécialité. Le dossier est pratiquement le même lors de la réinscription, auquel viennent s'ajouter des documents permettant à l'autorité responsable d'évaluer l'expérience acquise entre-temps. Les conditions d'inscription et de réinscription sur la liste nationale sont fixées par l'article 17 du décret $n^{\circ}$ 2004-1463 du 23 décembre 2004. Le candidat qui veut être inscrit ou réinscrit sur cette liste doit envoyer son dossier, également avant le 1er mars, au procureur général près la Cour de cassation, lequel étudie ensuite sa demande. Conformément à la loi, la Cour de cassation doit vérifier la durée d'inscription sur une liste près la Cour d'appel. Personne ne peut être inscrit sur la liste nationale sans être d'abord inscrit sur la liste tenue par une Cour d'appel. Le procureur général près la Cour de cassation recueille l'avis du premier président et du procureur général près la Cour d'appel où le candidat est inscrit et transmet ensuite la candidature, avec son avis, au bureau de la Cour de cassation. En France, il existe donc deux registres d'experts judiciaires. Chaque Cour d'appel régit sa propre liste d'experts judiciaires ; il existe ainsi 35 listes près des Cours d'appel. Le candidat est inscrit pour trois ans à titre probatoire et, à l'issue de cette période, peut être réinscrit pour une durée de cinq ans. La Cour de cassation, juridiction suprême de France, est la seule juridiction à détenir la liste nationale. Tout expert judiciaire briguant une intégration sur la liste nationale, pour une durée de sept ans avec renouvellement éventuel, doit pouvoir démontrer son inscription depuis un minimum de cinq ans sur la liste d'une Cour d'appel. Les experts judiciaires doivent, selon l'article 22 du décret n²004-1463 du 23 décembre 2004, prêter serment auprès de la juridiction agréée. Cet article fixe également les conditions concernant les personnes morales : le serment est prêté par leur représentant, désigné à cet effet. Le compte rendu annuel de l'activité récapitule toutes les missions qui ont été ordonnées par les magistrats ou par les officiers de police judiciaire. II doit être envoyé au premier président et au procureur général de la Cour d'appel ou de la Cour de cassation, selon le cas. La rémunération du traducteur et/ou interprète assermenté est fonction de la nature de la procédure. Pour ce qui est des missions judiciaires civiles et non judiciaires, la tarification est libre et dépend donc du traducteur. Pour les missions de procédure pénale et de procédure administrative, une page de traduction (soit 250 mots français) est payée 25 euros et une heure d'interprétation 30 euros.

L'activité des traducteurs-interprètes assermentés en République tchèque est toujours régie par la Loi n 36/1967, soumise ultérieurement à diverses modifications. Le décret du ministère de la Justice $n^{\circ} 37 / 1967$ relatif à l'application de la Loi sur les experts et les interprètes a aussi subi plusieurs remaniements (décrets n 11/1985, 184/1990, 77/1993 et 432/2002). La réglementation a été ajustée en raison d'une définition insuffisante du contrôle par l'administration du travail des traducteurs/interprètes, de la mauvaise qualité de certaines traductions, du non-respect des délais et de la confidentialité et du refus de réaliser traductions et 
interprétations pour l'administration. Les conditions à remplir pour être nommé interprète assermenté sont devenues plus contraignantes, les fautes répréhensibles commises par les interprètes sont plus clairement définies (comme en France), tout comme la possibilité de suspendre ou d'annuler l'inscription d'un interprète sur la liste officielle. La législation tchèque - à l'instar de la réglementation espagnole - ne distingue pas le métier de traducteur du métier d'interprète et emploie le terme d'interprète pour désigner les traducteurs-interprètes assermentés. La nouvelle loi slovaque, quant à elle, distingue clairement l'activité de traduction de l'activité d'interprétation et fait coexister deux listes officielles distinctes. En Slovaquie, il est donc possible de demander l'inscription sur une seule ou sur les deux listes. En République tchèque, l'interprète assermenté est nommé par le Tribunal régional de son domicile. II n'existe qu'une seule liste d'experts et d'interprètes assermentés, tenue par le ministère de la Justice. Pour souhaiter devenir interprète assermenté, il faut tout d'abord : i) être citoyen de la République tchèque, ii) être citoyen d'un autre pays de l'Union européenne et justifier d'une résidence permanente ou temporaire en République tchèque, ou iii) être citoyen d'un pays non membre de l'Union européenne et justifier d'une résidence permanente en République tchèque. En parallèle, il est nécessaire d'être titulaire d'un Master (idéalement en traduction et interprétation, ou en philologie), de suivre le cours pour interprètes et traducteurs organisé par la faculté de droit (terminologie juridique tchèque et séminaire linguistique), et d'exercer depuis au moins cinq ans le métier de traducteur et/ou d'interprète. II n'y a donc aucun examen et la décision dépend exclusivement du Tribunal régional compétent. Autre condition, facultative : suivre un cours organisé par la Chambre des traducteurs assermentés. La rémunération des interprètes est définie par le décret $n^{\circ}$ 432/2002. Une page de traduction (à savoir 30 lignes de 60 caractères) et une heure d'interprétation sont payées de 100 à 350 couronnes tchèques (de 4 à 14 euros) en fonction des exigences du travail. L'interprète assermenté tchèque doit, une fois par an, rendre compte de ses activités au Tribunal régional qui l'a nommé - à l'instar de son homologue français.

\section{Comparaison}

En France et en République tchèque, les interprétations et traductions assermentées relèvent de la compétence du ministère de la Justice. En Espagne, ces activités relèvent de la compétence du ministère des Affaires étrangères et de la Coopération, ce qui aujourd'hui ne répond plus à aucune logique ni pratique et ne reflète plus les besoins ni le travail exercé par les traducteurs-interprètes jurés dans ce pays. La traduction assermentée a été réglementée en République tchèque en 1967, en France en 1971, en Espagne en 1977. La réglementation a ensuite été modifiée plusieurs fois dans les trois pays. La législation française relative à la traduction-interprétation assermentée est très complexe. À la différence de l'Espagne où le traducteur-interprète juré n'est pas tenu de rendre compte de ses activités à l'administration, 
son homologue tchèque ou français est subordonné à la justice et la réglementation fixe sa responsabilité pénale. Le traducteur-interprète juré en Espagne et l'interprète assermenté en République tchèque sont nommés pour une durée indéterminée, tandis qu'en France il faut périodiquement déposer une nouvelle candidature pour maintenir son inscription sur la liste. En France et en République tchèque, il n'y a pas d'examen pour devenir traducteur et/ou interprète assermenté. Les examens en Espagne sont souvent critiqués parce qu'ils ne correspondent pas au travail des traducteurs/interprètes jurés en situation réelle et ne sont pas uniformes. Ainsi, de nos jours, il est pratiquement impossible de réaliser des traductions sans équipement informatique, connexion internet et supports disponibles en ligne, contrairement à ce que requiert l'examen. De plus, dans les deux premières parties de la deuxième épreuve, il est interdit d'utiliser un dictionnaire, pourtant autorisé dans la dernière partie. Mais c'est la troisième épreuve (interprétation consécutive) qui est la principale cible des critiques, car elle ne permet en aucun cas une évaluation sérieuse des connaissances et des aptitudes nécessaires à l'interprète professionnel. Globalement, toutefois, le système espagnol, basé sur des examens, est plus objectif que les systèmes français et tchèque, qui se focalisent sur l'expérience professionnelle des candidats et dépendent de Cours de justice dont les critères d'évaluation des candidatures diffèrent. Le système de nomination en République tchèque et en France est donc subjectif et n'élimine pas le risque de corruption, tandis qu'en Espagne, tout candidat ayant réussi les examens peut être inscrit sur la liste des traducteurs-interprètes jurés : ce n'est pas la législation, mais plutôt la pratique et les habitudes qui définissent le travail de ces traducteurs-interprètes. Les normes juridiques ne fixent que l'accès au métier et certains aspects essentiels de sa pratique, tels que les exigences minimales pour que la traduction ait une valeur officielle. Par contre, en France, la réglementation définit explicitement toutes les questions liées aux traductions assermentées, y compris les honoraires et les éventuelles sanctions en cas de travail mal fait.

De toute évidence, en République tchèque, la loi, adoptée il y a cinquante ans sous le régime communiste, ne correspond plus à la situation juridique et économique actuelle. Depuis 1989, les juristes et les traducteurs discutent et demandent la promulgation d'une nouvelle loi, mais aucun projet de loi n'a été approuvé à ce jour et ce texte archaïque est toujours en vigueur (à la différence de la Slovaquie qui l'a abrogé et remplacé en 2004 par une nouvelle loi, mieux adaptée à la situation contemporaine). La République tchèque ne distingue pas, contrairement à la France, le métier de traducteur assermenté du métier d'interprète assermenté, bien qu'il s'agisse de deux activités distinctes, exigeant des compétences complètement différentes. L'Espagne distingue ces métiers, mais il est impossible d'être nommé uniquement traducteur juré ou interprète juré parce qu'il n'existe, comme en République tchèque, qu'une seule liste de traducteurs-interprètes jurés. En République tchèque, où il n'y a pas d'examen en interprétation (ni en traduction), on peut trouver sur la liste des interprètes assermentés nombre d'experts qui refusent les interprétations parce qu'ils ne maîtrisent pas suffisamment la langue étrangère et ne réalisent que des traductions. Cette distinction entre interprètes et traduc- 
teurs assermentés peut également comporter des aspects négatifs. En Slovaquie, on observe ainsi que les interprètes assermentés sont beaucoup moins nombreux que les traducteurs assermentés, ce qui peut s'expliquer - outre les raisons déjà mentionnées - par le fait que certains experts ont une autre activité professionnelle en parallèle (surtout dans l'enseignement), ce qui les empêche d'exercer en tant qu'interprètes.

En France et en République tchèque, les traducteurs-interprètes assermentés sont nommés par la justice : en France par le président d'une Cour d'appel ou de la Cour de cassation, en République tchèque par le président d'un Tribunal régional. En République tchèque, les candidats peuvent déposer leur dossier à tout moment de l'année et les nominations ont lieu en général deux fois par an, tandis qu'en France, il faut soumettre sa candidature à date fixe. Seules les personnes physiques peuvent être nommées en République tchèque, tandis qu'en France, la nomination des personnes morales est possible. En République tchèque, chacun des huit Tribunaux régionaux administre sa liste d'experts. La liste nationale représente la somme de ces listes régionales et elle est tenue par le ministère de la Justice. En France, chacune des trente-cinq Cours d'appel gère sa propre liste, mais la liste nationale, tenue par la Cour de cassation, est complètement indépendante : elle ne reprend pas les listes des Cours d'appel et comporte exclusivement des noms d'experts retenus par la Cour de cassation ellemême, ce qui peut sembler très opaque et compliqué. II existe également en France depuis 2005 une limite d'âge, fixée à soixante-dix ans, pour exercer le métier de traducteur et/ou d'interprète assermenté, et, alors qu'en République tchèque seule une expérience professionnelle de cinq ans est exigée avant la nomination, en France, l'expérience professionnelle n'est pas quantifiée, elle doit simplement être "suffisante ". L'absence d'examen et la nomination à vie en République tchèque aboutissent à une liste d'experts dont beaucoup ne sont plus actifs (deux tiers des inscrits selon les estimations). L'existence d'examens en Espagne a réduit considérablement le nombre de traducteurs jurés, mais, en parallèle, a augmenté la qualité des prestations. C'est aussi le cas en Slovaquie, où le gouvernement a introduit des examens.

Après sa nomination, l'interprète assermenté en République tchèque reçoit le journal de l'interprète, dans lequel il enregistre toutes les traductions et interprétations réalisées. II obtient aussi la carte d'interprète qui lui permet de faire apposer le cachet officiel. Pour sa part, le traducteur et/ou interprète assermenté en France obtient simplement le cachet officiel et est tenu, chaque année, d'envoyer à la Cour le récapitulatif de ses activités. En France - à la différence de la République tchèque - la formation continue des experts étant obligatoire, il joint aussi la liste des modules de formation suivis. En Espagne et en France, il est recommandé de souscrire une assurance de responsabilité professionnelle, ce qui n'est pas le cas en République tchèque, alors qu'en Slovaquie cette assurance est obligatoire. 
Jaromír Kadlec est traducteur assermenté (français et espagnol vers le tchèque) nommé par le Tribunal régional d'Ostrava et maître de conférences (traductions et français de spécialité) à l'Université Palacký d'Olomouc en République tchèque.

\section{Bibliographie}

CHAUVAUD Frédéric, 2003, Experts et expertise judiciaire, France, XIXe et xxe siècles, Rennes (France), Presses Universitaires de Rennes.

FUSILIER Évelyne, 2010, "Traducteurs et interprètes experts : une exception française ? ", Traduire, $n^{\circ} 223$, p. 8-37.

GALANES SANTOS Iolanda, 2010, "La acreditación de traductores y/o intérpretes jurados en España: novedades, contrastes e incoherencias ", Sendebar, n² 21, p. 251-270.

HONOVÁ Zuzana, 2011, "La position actuelle de l'interprète assermenté en République tchèque ", Studia Romanistica, n 11/1, p. 109-117.

LOBATO PATRICIO Julia, 2010, "Réalité professionnelle de la traduction assermentée en Espagne ", Traduire, n²23, p. 49-65.

PEÑAROJA FA Josep, 2013, "Histoire des experts traducteurs et interprètes ", Traduire, $\mathrm{n}^{\circ} 228$, p. 121-134.

VIGIER MORENO Francisco, 2010, "La nueva normativa de la profesión de traductor-intérprete jurado: ¿un paso adelante o un paso atrás? ", La Linterna del Traductor, n 4, p. 37-49. 